\title{
La pratique chez Patočka : une description phénoménologique de l'appartenance
}

\section{Charles-André Mangeney}

\section{(2) OpenEdition}

1 Journals

Édition électronique

URL : http://journals.openedition.org/alter/636

DOI : $10.4000 /$ alter.636

ISSN : 2558-7927

Éditeur :

Association ALTER, Archives Husserl (CNRS-UMR 8547)

\section{Édition imprimée}

Date de publication : 31 décembre 2018

Pagination : 177-196

ISBN : 978-2-9550449-4-0

ISSN : 1249-8947

\section{Référence électronique}

Charles-André Mangeney, «La pratique chez Patočka : une description phénoménologique de l'appartenance », Alter [En ligne], 26 | 2018, mis en ligne le 31 décembre 2019, consulté le 23 mars 2020. URL : http://journals.openedition.org/alter/636 ; DOI : https://doi.org/10.4000/alter.636 


\title{
LA PRATIQUE CHEZ PATOČKA : UNE DESCRIPTION PHÉNOMÉNOLOGIQUE DE L'APPARTENANCE
}

\author{
Charles-André Mangeney
}

Les investigations phénoménologiques de Patočka ne prennent leur sens que si elles sont rattachées à un dilemme coextensif à la phénoménologie elle-même. Il est en effet possible d'affirmer que la phénoménologie trouve son sens originaire dans une révolte contre l'univocité de l'être ${ }^{1}$, et ceci dès sa fondation par Husserl. Sa critique du psychologisme et du naturalisme n'a $\mathrm{qu}^{\prime}$ un sens profond: montrer qu'être ne signifie pas partout la même chose, ce que Husserl s'emploie à faire en distinguant l'ontologie de la région chose de l'ontologie de la région conscience ${ }^{2}$. Ainsi, la conscience qui fait apparaître le monde (comme totalité des choses) ne peut pas elle-même être une "parcelle de monde $»^{3}$, c'est-à-dire tomber dans le monde et avoir le sens d'être d'une chose. C'est pourquoi l'existence humaine présuppose toujours une rupture d'appartenance. En un mot, pour Husserl, comme pour Heidegger et Scheler, respecter le mode d'être propre de la conscience, c'est la démondanéiser - «démondanéiser » ne doit pas être compris ici au

\footnotetext{
${ }^{1}$ La question de l'univocité de l'être doit être rapportée au problème, d'ascendance aristotélicienne, du statut logique de l'être : doit-on dire que l'être est un genre et qu'il sert donc d'essence à tout ce qui est, ou au contraire que l'être ne sert d'essence à aucune chose (« tó $\delta$ '

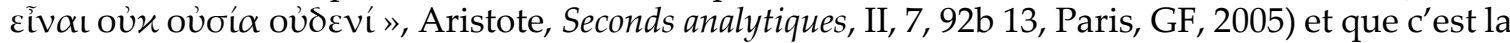
différence ontologique des modes d'être qui est première ? Sur ce point décisif, voir P. Aubenque, Le problème de l'être chez Aristote. Essai sur la problématique aristotélicienne. Paris, PUF, 2013, p. 222. 2 Levinas, dans sa thèse sur Husserl, a très bien vu que le naturalisme et le psychologisme sont porteurs d'une thèse commune sur l'être : être, c'est être dans la nature, et par conséquent la conscience doit être un contenant physique dans lequel s'enchaînent des contenus inertes selon les lois de la nature (temps, espace, causalité et action réciproque). Voir E. Levinas, La théorie de l'intuition dans la phénoménologie de Husserl, Paris, Vrin, 2010, p. 33.

3 « [...] on ne devra penser à aucun titre que, dans notre moi pur apodictique, nous ayons réussi à sauver une petite parcelle de monde [...]», E. Husserl, Méditations cartésiennes. Introduction à la phénoménologie, Paris, Vrin, 1992, p. 51 (nous soulignons).
} 
sens d'un anéantissement du monde, tel qu'on peut le trouver par exemple chez Hobbes dans le De Corpore ou même chez Descartes ; il s'agit simplement d'affirmer une équivocité de l'être impliquant une incommensurabilité radicale de l'être de l'homme ou de la conscience et de l'être du monde, ou des choses: démondanéisation non pas comme isolement de la conscience sur le plan de l'existence, mais sur le plan de l'essence : être pour l'homme ne signifiant pas alors la même chose qu'être pour le monde - et lorsque Patočka écrit que « le rapport humain à la totalité signifie qu'il y a chez l'homme une incomplétude essentielle, une révolte contre la totalité, une dissension avec elle. La vie humaine est à vrai dire une vie contre la totalité ${ }^{4}$, il se place directement dans cet héritage phénoménologique.

Patočka écrira cependant, quelques années plus tard «[...] que le problème du monde implique qu'une partie de l'étant se rapporte à l'étant en totalité $»^{5}$. Il semble ainsi précisément se placer du côté de la critique merleau-pontienne des fondateurs de la phénoménologie. Cette critique s'efforce de montrer qu'au contraire, ce n'est que sur fond de parenté ontologique (et donc d'univocité de l'être) entre le sujet et le monde, que ce dernier peut apparaître. Il faut que nous soyons pris dans le tissu du monde pour que celui-ci se manifeste à nous, et c'est précisément parce que nous sommes chose parmi les choses (c'està-dire corps) ${ }^{6}$ que celles-ci peuvent se donner (aucune perception n'est imaginable sans un corps propre depuis lequel elle a lieu $)^{7}$ : nous $\mathrm{n}^{\prime}$ avons donc de rapport si intime avec le monde, avec l'étant, nous ne pouvons nous projeter intentionnellement en lui que si nous en sommes déjà, que grâce à une communauté à laquelle nous appartenons toujours déjà. Nous retrouvons ici le sens d'un jeu de mot que l'allemand permet à Heidegger d'effectuer : on ne peut entendre, c' est-

\footnotetext{
${ }^{4}$ J. Patočka, Le monde naturel et le mouvement de l'existence humaine, Dordrecht/Boston/Londres, Kluwer Academic Publishers, 1988, p. 112.

${ }^{5} \mathrm{~J}$. Patočka, Le monde naturel comme problème philosophique, « Le monde naturel dans la médiation de son auteur trente-trois ans après », Paris, Vrin, 2016, p. 170.

${ }^{6}$ Ce que nous voulons dire ici, ce n'est pas que notre corps propre et vécu se réduirait au corps inerte et saisi du dehors qu'est le corps objectif, mais plutôt que pour que notre corps propre puisse percevoir les corps objectifs extérieurs, il faut pourtant qu'il y ait quelque chose de commun entre eux : ce n'est qu'au sein de la parenté qui unit tous les corps qu'un corps propre pourra se distinguer d'un corps objectif. Comme le dit Merleau-Ponty: " Le corps nous unit directement aux choses par sa propre ontogénèse, en soudant l'une à l'autre [...] la masse sensible qu'il est et la masse du sensible où il naît par ségrégation, et à laquelle comme voyant, il reste ouvert » (M. Merleau-Ponty, Le visible et l'invisible, Paris, Gallimard, « Tel », 1964, p. 183).

${ }^{7}$ On retrouve ici les fondements de l'intuition de Merleau-Ponty : le corps ne peut faire apparaître les choses que s'il en est d'abord une : «Visible et mobile, mon corps est au nombre des choses, il est l'une d'elles, il est pris dans le tissu du monde et sa cohésion est celle d'une chose ». M. Merleau-Ponty, L'cil et l'esprit, Paris, Gallimard, 1964, p. 19.
} 
La pratique chez Patočka : une description phénoménologique...

à-dire être à l'écoute (Hören auf...), que de ce à quoi on appartient (Gehören).

Faut-il penser ici que Patočka se contredit, qu'il soutient deux membres contradictoires d'une même alternative ? Nous pensons, bien plutôt, qu'il affronte là un dilemme central de la phénoménologie : ou bien sauvegarder le mode d'être propre de la conscience et de l'existence, en tant qu'elle est intentionnelle et qu'elle se projette dynamiquement en avant d'elle-même, mais perdre l'appartenance fondamentale du sujet au monde, ou bien au contraire réinscrire le sujet, en tant que corporéité motrice, dans le monde, mais risquer de perdre son sens d'être propre. La thèse que nous entendons soutenir dans ce travail est que Patočka, en soutenant à la fois les deux membres de cette alternative, ramène ce dilemme à une simple aporie, à un problème mal posé. Nous pensons que Patočka parvient en réalité à penser une existence irréductible à tout autre mode d'être, mais depuis l'appartenance du sujet au monde (dissociant ainsi appartenance et réification), et ceci grâce à la description d'une attitude intentionnelle inédite que nous nous proposons d'appeler pratique. En effet, et nous y reviendrons, la pratique permet de penser un sujet qui continue à vivre au devant de lui-même, ce qui le rend incommensurable aux choses en leur inertie, tout en pensant une rétro-action de la réalisation du projet sur le projet lui-même, c'est-à-dire un approfondissement et une altération de la possibilisation existentielle par sa réalisation même, ce qui implique une parenté ontologique du sujet et du monde, puisqu'il faut que notre commerce avec les choses puisse modifier la teneur du lien qui nous unit à elles, et qu'il faut donc que quelque chose du monde puisse passer en nous. Dans une première partie, nous reviendrons sur la confusion fondamentale qui grève les analyses de la phénoménologie classique (identifier appartenance et réification), puis nous montrerons comment Patočka s'en défait, en restituant les grandes lignes de la description phénoménologique de la pratique qu'il effectue.

\section{I. Être dans le monde ou être au monde : sauver le sens d'être du sujet au prix d'une confusion phénoménologique}

\section{L'héritage de Husserl, Heidegger et Scheler: la dé-réification de la conscience par la dislocation de son appartenance au monde}

Il ne sera possible de soutenir que Patočka place le sujet dans une position de porte-à-faux ontologique entre son sens d'être spécifique et

8 M. Heidegger, Questions I et II, Paris, Gallimard, « Tel », 1968, p. 265. 
son appartenance nécessaire au monde ${ }^{9} \mathrm{qu}^{\prime}$ aussi longtemps que nous demeurerons prisonniers des présupposés de la tradition phénoménologique qui constituent cette alternative déchirante. En un mot, affirmer qu'il y a une tension, voire une contradiction au cœur de la philosophie de Patočka, c'est manquer la profondeur de sa remise en cause de l'héritage husserlien, schélérien et heideggérien.

En quoi subjectivité et appartenance s'excluent-elles de prime abord ? Et avant cela, qu'entend-on communément par " appartenance » ? Il nous semble nécessaire de différencier en premier lieu inclusion et appartenance. L'inclusion désigne une relation d'extériorité réciproque : la boule qui se trouve dans son urne demeure indépendante, en son être, à l'égard de l'urne qui la contient. C'est-à-dire que le contenant, bien qu'il inclut en lui le contenu, ne l'altère pas. De même, je peux bien me retrouver dans la foule sans y appartenir, c'est-à-dire sans que cette inclusion n'ait aucune effectivité sur moi, si bien que l'on peut dire qu'avec la relation d'inclusion l'élément inclus demeure extérieur à cela qui l'inclut. L'appartenance, au contraire, instaure une relation d'intériorité réciproque, c'est-à-dire de dépendance mutuelle : l'être du tout dépend de l'être de ses parties, comme l'être des parties dépend de l'être du tout auquel elles appartiennent. Par exemple, appartenir à un groupe, c'est à la fois devoir ce que nous sommes au groupe qui nous enveloppe et constituer ce groupe en son être si bien que sans les êtres qui y appartiennent, celui-ci n'est pas conforme à son essence. C'est comme si chaque membre était le tout du groupe, alors même que le groupe a besoin, pour exister, de la totalité de ses membres. Appartenir, c'est donc ne pas pouvoir être séparé de ce à quoi on appartient sans perte radicale et dégradation de ce que nous sommes (on peut très bien quitter ce à quoi l'on appartient sans s'en séparer, mais la séparation est toujours un déchirement, et si elle n'est pas assumée, elle est un exil). On peut ainsi soutenir que Dufrenne décrit le rapport de la ville et du citadin comme un rapport d'appartenance : «[...] les hommes produisent leur ville comme la ville produit ses hommes, sans qu'aucun des partenaires puisse se targuer d'une initiative ou d'une priorité $»^{10}$. Appartenance signifie donc dépendance ontologique réciproque.

$C^{\prime}$ est une telle réciprocité que la phénoménologie, dès ses fondements, ne peut accepter. En effet, Husserl écrit : « qui nous sauve d'une

\footnotetext{
${ }^{9}$ Comme c'est le cas de Karel Novotný dans La genèse d'une hérésie, Monde, corps et histoire dans la pensée de Jan Patočka qui soutient qu'existe chez Patočka une «[...] dualité des perspectives : l'une qui porte sur la vie dans le monde donné, l'autre qui thématise son dépassement par le mouvement de l'esprit, correspondant à la double originarité de la donation du monde, effectuée à travers le corps vivant, et de la donation de la compréhension de l'être par l'esprit » (Paris, Vrin, 2012, p. 95).

${ }_{10}$ M. Dufrenne, dans la préface de P. Sansot, Poétique de la ville, Paris, Payot, 2004, p. 12.
} 
La pratique chez Patočka : une description phénoménologique...

réification de la conscience est le sauveur de la philosophie [...] $\gg^{11}$. L'intentionnalité, qui n'est pas une propriété de la conscience qui ellemême n'est ni une chose ni une relation entre les choses, devait être le moyen d'un tel sauvetage. La conscience est intentionnelle, c'est là sa façon d'exister, et en tant que pur se-porter-vers... elle est en ellemême un « se transcender » vers ce qui n'est pas elle, irréductible à tout étant qui serait d'abord avant d'entrer en relation avec autre chose ensuite. C'est pour préserver ce mode d'existence spécifique que Husserl croit bon de poser une extra-mondanéité de la conscience, sans quoi elle entrerait en relation réciproque et causale avec les autres choses et tomberait alors dans le monde pour perdre son autonomie absolue. C'est ce que signifie la fonction de constitution transcendantale de la conscience : afin qu'il ne soit plus possible de confondre la conscience avec un étant ou une production mondaine, c'est la conscience qui par ses prestations subjectives doit lui donner son sens ; et pour constituer cette totalité, elle ne doit pas en être. Si l'épochè telle que Husserl la décrit est possible, c'est-à-dire si la conscience peut se retourner sur l'ensemble de ses vécus de constitution du sens du monde et les objectiver, c'est-à-dire siéger «[...] au-dessus de son être naturel, et au-dessus du monde naturel $\gg^{12}$, elle ne peut être de ce monde. Scheler donnera à cette idée sa formulation décisive, en opposant l'esprit (Geist) à l'âme (Seele), en tant que l'esprit est une puissance d'objectivation de soi et du monde, ainsi que la capacité conséquente d'en disposer, et donc l'obligation d'en avoir la charge, alors que l'âme, que nous avons en commun avec tous les vivants, est la structure caractéristique du comportement qui témoigne de notre appartenance à la nature (phusis), ce qui fait de nous des êtres dotés d'une signification vitale. Scheler demeure absolument conséquent lorsqu'il affirme que cette capacité de recul (l'esprit) à l'égard de tout donné implique que son foyer n'ait pas de lieu, qu'il soit hors du monde: "Mais ce centre, d'où l'homme accomplit les actes par lesquels il "objective" le monde, son corps et son âme, ne peut pas être lui-même une partie de ce monde [...] Ainsi l'homme est-il l'être supérieur à soi-même et au monde $»^{13}$. Si le monde est bien ainsi la charge de l'homme, ce dernier demeure pour le monde hors d'atteinte.

Si Patočka reprend et discute Scheler et Husserl, c'est surtout par l'intermédiaire de Heidegger qu'il va faire sienne cette exigence

\footnotetext{
${ }^{11}$ Inédit de 1920, cité par F. Dastur, La philosophie allemande, Paris, PUF, 1993, p. 292.

12 E. Husserl, La crise des sciences européennes et la phénoménologie transcendantale, Paris, Gallimard, 1976, p. 172.

${ }^{13}$ M. Scheler, La situation de l'homme dans le monde, Paris, Aubier, 1951, p. 53.
} 
phénoménologique de préservation du sens d'être propre de la subjectivité et de la non-appartenance qu'elle implique. Patočka cite explicitement Heidegger : «Vivre, c'est réaliser des possibilités. La réalisation de possibilités signifie donc, comme dit Heidegger, que la vie est toujours en avant d'elle-même, que lui appartient essentiellement le pro-jet ${ }^{14}$. Or, si pour Heidegger le Dasein est un être de projet, qui est sur le mode de ses propres possibilités, c'est d'abord parce qu'il n'est pas immédiatement quelque chose, mais qu'il peut prendre $\mathrm{du}$ recul sur son être et l'être du monde, pour les interroger (il n'est pas mais $a$ à être). Mieux, c'est parce qu'il est ce recul, cette constante possibilité de néantisation (Vernichtung) ${ }^{15}$ même qu'il peut être ouverture à l'être. Ce privilège insigne de pouvoir poser la question de l'être est la conséquence directe d'une désolation et d'un déracinement fondamental. Le Dasein est d'abord défini par le "comprendre» (Verstand), par lequel il fend les choses et le présent pour se projeter vers l'être qu'il a à être dans l'avenir, et c'est par ce comprendre, qui est d'abord un secomprendre, que le Dasein donne sa significativité (Bedeutsamkeit) au monde. Ainsi, les étants autour de moi ne s'organisent en une totalité signifiante, c'est-à-dire ne se donnent comme une totalité de renvois vivants, que parce que, en tant que Dasein, j'ai auparavant ouvert le monde par le projet de mon être. On dira donc que le Dasein n'est pas dans le monde, mais qu'il est au monde, au sens où il dépasse les étants pour leur donner un sens à partir du projet qu'il a pour lui-même ${ }^{16}$. Pour le dire avec Heidegger, l'ensemble des « quoi » (des sens conférés aux étants par renvois à ce qu'ils peuvent) s'origine dans un «quoi » qui ne renvoie plus à rien, et qui est "qui », un projet d'être posé par le Dasein en sa transcendance. C'est donc le Dasein qui, en tant qu'il n'est pas, décide du sens de ce qui est. On appellera alors authenticité (Eigentlichkeit) le mouvement par lequel le Dasein, se réappropriant pour lui-même sa liberté de décider du sens du monde, va par sa résolution (Entschloßenheit), se donner de nouvelles possibilités, possibilités qui sont proprement les siennes, dont il est l'auteur en personne, par lesquelles il pourra se comprendre soi-même et le monde en original : c'est « [...] l'être-pour le pouvoir-être le plus propre, c'est-à-

\footnotetext{
14 J. Patočka, "Leçons sur la corporéité », Papiers phénoménologiques, Grenoble, Jérôme Millon, 1995, p. 92.

${ }^{15}$ La Vernichtung lui permettant d'interroger, suppose qu'il ne dépende, n'appartienne à rien, pour poser la question générale du sens de l'être : M. Heidegger, Être et temps, trad. E. Martineau, $\S 4$, p. 31 (édition numérique).

${ }_{16}$ «Le Dasein est [...] la condition ontique de possibilité de la découvrabilité de l'étant qui fait encontre dans un monde sur le mode de la tournure [...] », ibid., § 18, p. 87.
} 
La pratique chez Patočka : une description phénoménologique...

dire l'être-libre pour la liberté du se-choisir-et-se-saisir-soi-même $»^{17}$. On voit donc que cette ouverture au monde qu'est la condition ontologique du Dasein n'est possible que parce qu'il est libre à son égard, qu'il $\mathrm{n}^{\prime}$ est donc pas pris en lui. La tradition phénoménologique se fonde donc sur un présupposé très net: la confusion de l'appartenance au monde et de la réification ( $c^{\prime}$ est-à-dire de la perte du sens d'être) de la conscience.

Il est donc troublant de voir Patočka reprendre la description heideggérienne de l'existence au-devant de soi du Dasein, et même son concept d'authenticité18 qui implique une liberté maximale à l'égard du monde, en même tant que l'affirmation selon laquelle l'homme est une partie de l'étant se rapportant à l'étant en totalité. Comment est-ce possible?

\section{Une conséquence majeure: un intellectualisme au cour de la} description phénoménologique de l'existence

Ce double versant de la philosophie de Patočka n'est un problème qu'à la condition de penser qu'il reprend la totalité de l'analytique existentiale (et donc les présupposés que nous venons de mettre en lumière). Or, nous allons maintenant montrer que Patočka organise une réfection bien plus profonde de l'analytique du Dasein que ne le pensent les commentateurs ${ }^{19}$.

En effet, l'idée que le Dasein est libre à l'égard du monde se traduit par un intellectualisme qui prend la figure suivante : il s'agit de la séparation absolue de la conception et de la réalisation. Ce que nous qualifions d'intellectualisme n'est pas tant le fait qu'il y ait de la conception, mais à la fois la séparation de la conception et de la réalisation, et le primat donné à la conception, que la conception soit le principe de l'action. Cela vaut pour n'importe quelle occupation « pratique » au sens trivial du terme: l'intellectualisme dont nous parlons suppose que pour bien sculpter une forme dans le bois, par exemple, il suffirait de l'avoir bien conçue, d'en avoir bien imaginé par avance le tracé. Or en réalité, il ne sera possible de concevoir une telle sculpture qu'au fur et à mesure du travail du bois. En d'autres termes, pour l'intellectualisme,

\footnotetext{
17 Ibid., § 40, p. 157.

${ }_{18}$ Puisque Patočka affirme que nous avons «[...] la possibilité de nous expliquer avec la possibilité que nous sommes justement et en totalité [...] » in Le monde naturel comme problème philosophique, op. cit., p. 241.

${ }^{19}$ Nous nous rangeons du côté de Renaud Barbaras, pour lequel Patočka parvient à une « déformalisation » de l'analytique existentiale, même si nous pensons qu'elle va plus loin que ce qu'il laisse entendre. Voir R. Barbaras, L'ouverture du monde, Lecture de Jan Patočka, Chatou, Les Éditions de la transparence, 2011, p. 116.
} 
le sens de l'existence réside tout entier dans l'intention, dans le projet ou le possible que le Dasein conçoit pour lui-même et projette de façon autonome. La liberté existentielle consiste précisément en la conception de nouvelles fins, par un effort d'extraction et de négation à l'égard des fins moyennes et communes, que nous n'avons pas choisies. Mais la réalisation de ces possibilités n'est pas pensée comme telle, elle se borne à être une exécution, un passage à l'existence comparable au passage à l'existence des cent Thalers dans l'exemple kantien et dont la venue au monde n'altère ni n'enrichit en rien leur conception. Le passage à la matière est tout négatif, c'est-à-dire que la confrontation des possibilités projetées par le Dasein à la situation de leur réalisation, au monde où elles doivent prendre corps, n'altère en rien leur contenu. Selon la distinction qu'opère Sartre entre la création et l'action, il faut dire que le Dasein est avant tout créateur, et non pas acteur:

L'action suppose un déterminisme [...] elle obéit à la nature pour lui commander [...] Mais la création est pure liberté; avant elle il n'y a rien, elle commence par produire ses propres principes, elle invente avant tout la fin ; par là elle participe à la gratuité de la conscience ; elle est cette gratuité voulue, repensée, érigée en but ${ }^{20}$.

Autrement dit encore, le Dasein trouve sa liberté dans sa capacité de projection, et le projet, en tant que simplement projeté, trouve en luimême et sans sortir de soi une forme d'achèvement et de perfection.

Comment ne pas relier cet intellectualisme phénoménologique et existentiel à une certaine conception des œuvres humaines, séparant par exemple les arts libéraux des arts mécaniques, et faisant de l'idée, de la conception, du projet, le seul vrai travail de l'artiste ? - il est vrai qu'il serait possible de faire un travail tout entier pour fonder ce rapprochement, et nous n'avons pas les moyens de le faire ici. Nous voudrions cependant insister sur le contenu commun de ces deux « intellectualismes » : dans les deux cas, il est question d'une prise effective sur les choses qui se serait construite loin des choses. L'artiste qui, suivant son idée, donne des ordres à ses assistants, voit le contenu artistique de son œuvre toute entier ramassé dans son projet, de même que le Dasein qui s'ouvre à son pouvoir-être propre par sa résolution voit son existence ramassée dans le projet qu'il a pour lui-même. Dans les deux cas, le sujet autonome donne sens au monde sans que ce sens luimême provienne du monde qu'il informe. Le génie est créateur comme le Dasein est authentique, c'est-à-dire, comme l'a bien montré Sartre,

${ }^{20}$ J.-P. Sartre, Baudelaire, Paris, Gallimard, 1947, p. 49. 
qu'il tire tout de lui-même, et le passage à la matière de son projet laisse ce dernier intact, comme la réalisation de l'idée de l'artiste est reléguée à un pur problème technique qui ne relève pas de son corps de métier ${ }^{21}$. Ainsi, de même que ce qui fait la valeur de l'œuvre se trouve tout entier dans sa conception, de même, le tout de l'existence du sujet se trouve dans la décision et la projection de ses possibilités. Cet intellectualisme phénoménologique, qui trouve sa source dans l'impératif de nonappartenance du sujet au monde, engendre lui-même la conséquence suivante : la phénoménologie, depuis ses début, n'a été capable de penser l'activité humaine que sous la forme de l'action, et non pas de la pratique (or l'action d'allumer une cigarette n'est pas équivalente à la pratique du piano).

Le propre d'une action, en ce qui concerne sa structure modale, c'est qu'elle commence et qu'elle s'achève tout entière avec sa possibilité. Une action menée à bien a été exécutée comme prévu, il faut que son passage à l'effectivité soit strictement identique à sa préfiguration irréelle. En toute rigueur, dans l'action, le possible donné et prédéfini épuise le réel (il n'y a rien de plus dans le réel que dans le possible, et l'action se ferme sur elle-même lorsque la réalisation a rejoint l'anticipation) : elle est totalement sous la dépendance du projet du sujet. Ainsi donc, si je sens une envie de fumer, que je la reprends à mon compte, je fais alors le projet d'allumer une cigarette (je m'élance vers un état du monde où ma cigarette est allumée). Une fois la cigarette allumée, mon projet s'étant refermé avec sa réalisation, je dois en ouvrir un autre (c'est-à-dire investir un nouveau point de fuite dans l'avenir etc.).

Or, tout travail accompli originairement et en première personne nous apprend qu'il est impossible de séparer le projet (ou la conception) de la réalisation, comme l'essentiel de l'in-essentiel, comme il n'est pas de vrai artiste qui ne soit en même temps artisan. Cela revient à dire que le projet seulement conçu ne peut se considérer comme achevé sans une rétro-action de sa réalisation sur lui-même : le projet ne peut se contenter de dépasser les étants vers une fin qu'il leur impose et qui est toujours déjà d'avance connue, mais il faut qu'il se découvre lui-même au fur et à mesure de sa réalisation. La fin du projet doit émerger progressivement à mesure qu'il se réalise, la réalisation s'insinue alors dans la conception et la féconde. C'est ce que soutient Valéry : «[...] il faut des mains, non seulement pour réaliser, mais pour

\footnotetext{
${ }^{21}$ On sait qu'à la Renaissance c'est l'atelier de l'artiste et non l'artiste lui-même qui est en charge de l'exécution de ses directives. Dürer, dans son Autoportrait à vingt-huit ans, portant un manteau avec col en fourrure, est peint sans instruments, etc.
} 
concevoir l'invention la plus simple sous forme intuitive $[\ldots] »^{22}$; en langage phénoménologique, cela signifie que la possibilisation du Dasein ne peut s'effectuer en amont du monde mais en lui - il semble que si le monde peut nous léguer ses possibilités, possibilités qu'au lieu de projeter nous nous contentons de révéler, il faut bien que nous ayons un corps et que ce corps soit une instance phénoménologique originaire de possibilisation : comment saurais-je ce que je peux faire d'un instrument de musique si mes doigts ou ma bouche ne découvraient pas au fur et à mesure de leur mouvement ce qui leur est possible de faire avec lui ? - et c'est cet approfondissement et cet affouillement du possible par sa confrontation au monde que nous nommons pratique.

Or, et c'est ce que nous allons montrer maintenant, Patočka décrit l'existence avant tout à l'aune de la pratique et non de l'action $^{23-}$ nous référons ici explicitement à l'opposition de l'action et de la pratique pour faire ressortir l'originalité de la description patočkienne du mouvement: l'action et la pratique sont deux types de mouvements qui ne peuvent être confondus et les interprètes de Patočka, à ne parler que du « mouvement » en général, ont manqué l'originalité des avancées phénoménologiques de Patočka en ce domaine - ce qui, en toute rigueur, implique qu'il ne peut pas se maintenir dans l'analytique existentiale sans la bouleverser en profondeur. En retraçant maintenant les linéaments de sa phénoménologie de la pratique, nous allons prendre la mesure des bouleversements qu'il fait subir à l'analytique du Dasein.

\section{Une phénoménologie de la pratique : une autre description de l'existence}

\section{Le sens originaire du dynamisme du mouvement : ses conditions de possibilité sont aussi ses résultats}

Pour montrer comment Patočka, en refusant le dualisme du sujet et du monde ou de la nature, parvient à rendre compte du sens d'être de la subjectivité depuis son appartenance au monde, nous devons partir

\footnotetext{
22 P. Valéry, « Discours aux chirurgiens », Variété III, IV et V, Paris, Gallimard, 1944, p. 588.

23 Précieuse est ici la typique des exemples dont Patočka se sert. Il est toujours question d'activités dans lesquelles il s'agit de séjourner et de s'installer pour les approfondir : « La pianiste virtuose, la dactylo parfaite, le joueur d'échecs ont fait un choix parmi plusieurs possibilités [...] », ou encore «Chacun sait que le fait de rédiger un article ou de se préparer à un examen [...] » et «Qu'il s'agisse de faucher de l'herbe ou de souffler du verre, chaque travail a sa propre hiérarchie de mouvements formant une unité », respectivement dans J. Patočka, Papiers phénoménologiques, op. cit., "Phénoménologie et métaphysique du mouvement », p. 25 et « Leçons sur la corporéité », p. 72.
} 
La pratique chez Patočka : une description phénoménologique...

du mouvement. Renaud Barbaras et Dragos Duicu ont bien montré que Patočka concrétisait l'analytique existentiale en soulignant le rôle de la corporéité et du mouvement dans la réalisation du projet du Dasein. Le sujet qui choisit ses possibilités et vit en elles, doit aussi pouvoir se mouvoir pour les effectuer : "Ce que je veux accomplir « en vue de mon être » est en réalité toujours co-déterminé par ce que je dois accomplir afin d'accomplir quoi que ce soit en général », et le corps sera alors « [...] l'ensemble des possibilités que nous ne choisissons pas, mais dans lesquelles nous nous insérons $[\ldots] \gg^{24}$. Le corps est ainsi la condition négative, le réquisit du mouvement, lui-même chargé d'effectuer les possibilités librement choisies ${ }^{25}$. En somme, Patočka se contenterait de lier l'existence $\mathrm{d} u$ Dasein à son soubassement physique réel.

Nous pensons qu'il va plus loin. S'il affirme bien que tout mouvement se réalise sur le fond $\mathrm{d}^{\prime}$ " [...] un subjectif pur, intentionnellement anticipé et projeté au préalable ${ }^{26}$ (sans intention qui le guide, mon mouvement est aveugle comme celui d'une pierre qui tombe), il écrit pourtant la chose suivante : "Contre Heidegger : il n y a aucune projection primaire de possibilités - le monde n'est pas un produit de la liberté, mais simplement ce qui rend possible une liberté finie $\gg^{27}$. Il n'y a donc pas d'activité projective : si le Dasein est bien sa propre ek-stase temporelle, il n'en n'est jamais le fondement ou le principe. Comment penser à la fois l'impossibilité d'une projection autonome de nos possibilités dans l'avenir, tout en maintenant en même temps que la vie du sujet est une vie dans des possibilités, et que tout mouvement n'est possible que sur le fondement d'une anticipation subjective, sans sombrer dans une contradiction? En réalité, Patočka se donne les moyens de penser l'un et l'autre, précisément parce qu'il sort du modèle de l'action. Il écrit que « [...] ce qui conditionne (la possibilité) ne peut être qu'un processus. La condition du processus n'échappe donc pas à la détermination "processus" ${ }^{28}$. Cela signifie que le mouvement emmène avec lui ses conditions de possibilité, et donc que la possibilité qui sous-tend le mouvement et lui donne son sens advient depuis ce

\footnotetext{
${ }^{24} \mathrm{~J}$. Patočka, Le monde naturel et le mouvement de l'existence humaine, op. cit., p. 94-96.

${ }^{25}$ Renaud Barbaras définira le corps comme «l'existential de l'effectivité du comprendre » (L'ouverture du monde, op. cit., p. 102) et Dragos Duicu distinguera alors les " possibilités libres » des " possibilités assumées" (Phénoménologie du mouvement. Patočka et l'héritage de la physique aristotélicienne, Paris, Hermann, 2014, p. 87).

${ }^{26}$ J. Patočka, "Phénoménologie et métaphysique du mouvement », Papiers phénoménologiques, op. cit., p. 19.

${ }^{27}$ J. Patočka, "[Corps, possibilités, monde, champ d'apparition]», Papiers phénoménologiques, op. cit., p. 122.

${ }^{28}$ J. Patočka, «Phénoménologie et ontologie du mouvement », Papiers phénoménologiques, op. cit., p. 42.
} 
mouvement lui-même et ne peut être envisagé que depuis sa réalisation.

C'est précisément dans la pratique que l'on trouve à la fois cette surrection du possible depuis le mouvement, en même temps que la préséance du possible sur le mouvement. En effet, dans la pratique se manifeste comme une disponibilité de la possibilité à un au-delà d'ellemême, à une relance (alors que dans l'action, on s'en souvient, le possible ouvre et ferme le mouvement). Si je souhaite par exemple m'initier à l'ébénisterie ou à la menuiserie, je débute dans cette pratique avec un certain nombre de possibilités rudimentaires et mal adaptées (je vis donc toujours déjà dans des possibilités) : je peux bien saisir la lime à bois ou le marteau, mais je ne saurai pas quoi en faire, ou plus exactement, je ne saurai pas ce que cela fait en première personne que de les utiliser correctement, et ces possibilités vont précisément s'affiner et s'enrichir au fur et à mesure de leurs réalisations (ainsi, bien que je vive déjà dans des possibilités, de nouvelles possibilités vont surgir depuis la réalisation des premières). Le propre de la pratique, c'est donc que la possibilité qui sous-tend le mouvement se creuse et s'affouille par sa réalisation même. Celui qui pratique voit naître de l'intérieur de sa pratique de nouvelles possibilités, $c^{\prime}$ est le principe même de l'exercice : l'exercice est une répétition qui n'est pas un retour à l'identique. Le musicien qui répète encore et encore une œuvre musicale ne refait jamais la même chose. La rétroaction de l'activité sur sa possibilité est un constant approfondissement. C'est là ce qui nous permet de résoudre la tension évoquée plus haut : d'un côté le sujet ne projette plus librement ses possibilités, elles surgissent et nous arrivent, elles sont comme décrochées depuis le mouvement, mais d'un autre côté, ce n'est toujours que depuis ce que je sais faire, depuis mes possibilités, que se lèvent de nouveaux possibles : ainsi, tout mouvement que je fais est précédé d'une possibilité qui l'anticipe bien qu'en même temps se dévoilent à moi des possibilités nouvelles par le truchement de sa réalisation. En toute rigueur, il faut dire que les conditions de possibilité du mouvement sont en même temps ses résultats (le possible précède le mouvement auquel il succède pourtant), ou, ce qui revient au même, que toute actualisation d'une possibilité porte en elle l'actualité d'une possibilisation, ou y contribue ${ }^{29}$.

\footnotetext{
${ }^{29}$ Par exemple, l'ébéniste va développer au contact du bois des cals aux mains : on pourrait croire que ces durillons constituent un obstacle pour le toucher, alors qu'au contraire ils le rendent plus précis et riche, en centrant le tact sur certains points de la main, comme un zoom tactile. Le travail du boit ouvre donc ce travail même à sa propre profondeur et fait émerger de nouvelles possibilités : repérer certaines qualités du bois, sa nodosité et sa texture, etc. Voir au sujet des cals R. Sennett, Ce que sait la main, la culture de l'artisanat, Paris, Albin Michel, 2010, p. 211.
} 
La pratique chez Patočka : une description phénoménologique...

Nous sommes ainsi en mesure de comprendre toute la richesse de l'affirmation suivante: "La transcendance du Dasein n'est pas un « projet » [...] elle est à la fois une ek-stase et un recueillement, aussi essentiels l'un que l'autre ${ }^{30}$. En effet, nous pouvons vivre ek-statiquement, tout en recueillant les possibilités dans lesquels nous dépassons notre présent, les possibilités que l'on recueille nous viennent toujours d'un effort pour réaliser des possibilités qui sont déjà nôtres (c'est là une activité couronnée de passivité que dit bien le concept de recueillement).

Patočka va ainsi tenter de déployer l'intuition fondatrice de sa nouvelle théorie de l'existence à travers plusieurs concepts. Citons d'abord: "Le "je fais" présuppose toujours un "je peux" et se déploie sur sa base. Je fais toujours ce que je peux, et le miracle du faire tient à ce qu'il excède ce "je peux" originaire » ${ }^{31}$. Patočka montre d'abord que toute réalisation, tout mouvement ( je fais », c'est-à-dire tout mouvement vécu de l'intérieur de son faire, tout mouvement réalisé à la première personne), repose au préalable sur un «je peux», sur une possibilité, qui lui-même est un «je sais faire »32, c'est-à-dire quelque chose comme une capacité acquise, une possibilité "décrochée » par les mouvements antérieurs. On retrouve ici l'antécédence de la visée sur le mouvement. Mais il ajoute que par la réalisation de la possibilité en question et au sein du faire, un miracle se produit : le faire va au-delà du pouvoir, va plus loin que ce qu'il laissait espérer, il se libère donc et dépasse sa condition. Patočka découvre ici la portée purement phénoménologique du concept de miracle (nous vivons, en première personne, l'apparition de possibilités comme des miracles) : un miracle est d'abord une intervention divine qui introduit un déséquilibre dans la série causale générale au sein de laquelle rien ne se crée, rien ne se perd, et tout se transforme. Mais phénoménologiquement, un miracle est une apparition inexpliquée et sans antécédent, une venue à l'être à partir de rien : c'est précisément ce qui est à l'oeuvre dans la pratique, puisque de nos possibilités surgit quelque chose qui n'y était contenu sous aucune forme, qui advient de surcroit, qui est un ajout pur, une nouvelle possibilité. Nous l'expérimentons tous pour nous-mêmes, lorsque nous pratiquons par exemple sans parvenir à progresser, que nous sommes comme englués dans nos possibilités encore trop restreintes et qu'un beau jour surgit comme par effraction et sans crier gare une nouvelle possibilité, inattendue et décisive, qui reconfigure l'en-

\footnotetext{
30 J. Patočka, Qu'est-ce que la phénoménologie ?, Grenoble, Jérôme Millon, 2002, p. 216.

31 J. Patočka, «Leçons sur la corporéité », Papiers phénoménologiques, op. cit., p. 71 (nous soulignons).

${ }^{32} \mathrm{Ibid} .$, p. 70.
} 
semble de notre pouvoir (ainsi par exemple du danseur qui comprend, de but en blanc et alors qu'il luttait auparavant avec son exécution, un pas de danse difficile). Ce « je fais » qui mord sur le « je peux », désigne exactement cette ek-stase et ce recueillement, que nous nommons pratique. Par ek-stase, il faut entendre ici le devancement par lequel nous vivons dans des possibilités qui nous appartiennent et par lesquelles nous donnons sens à une situation présente, et par recueillement, cet effort tendu vers la passivité (lors d'un deuil par exemple, nous parlerons de recueillement pour désigner l'effort des vivants pour ressentir l'absence du défunt, la tension visant à prendre la mesure de l'ampleur du chagrin qui les envahit). La pratique, c'est exactement le fait que toute ek-stase soit un recueillement: que notre effort pour cheminer vers une possibilité soit en même temps un effort pour en recevoir une autre. Nous approfondissons alors notre maîtrise du monde depuis cette maîtrise même : «Tout niveau atteint, toute capacité acquise, quelle qu'elle soit, a dû cependant d'abord être découverte; chacune présuppose, antérieurement à elle-même, une maîtrise $d u$ monde dont elle est la continuation $»^{33}$.

Patočka éclaire cette nouvelle vision de l'existence par une image. Il établit que le mouvement subjectif permet «[...] d'avancer continûment au sein du réel, comme l'on saute de pierre en pierre dans le lit $\mathrm{d}^{\prime}$ une rivière $»^{34}$. La métaphore $\mathrm{n}^{\prime}$ est pas anodine car chaque pierre est à la fois destination et point d'appui, donc fin et condition de possibilité du mouvement. Chaque saut, c'est-à-dire chaque mouvement, nous fait arriver à ce qui lui permettra de repartir, comme chaque mouvement, dans l'existence, nous dévoile de nouvelles possibilités à partir desquelles de nouveaux mouvements seront possibles. Il faut cependant distinguer fin et résultat (le résultat peut être obtenu sans avoir été escompté, alors que la fin doit être voulue) : or ici, si la fin du saut est précisément la nouvelle pierre, son résultat est l'ouverture à la possibilité d'un nouveau saut, d'un nouveau point de vue et mouvement. Il faut donc distinguer ce à quoi le mouvement aboutit et ce qu'il permet.

Il y a donc bien chez Patočka cette idée d'une avancée dans le réel par itération, où les conditions de possibilité du mouvement sont aussi ses résultats, c'est-à-dire où nos possibilités sont à la fois toujours conquises et déposées par un mouvement, et sont en même temps ce qui va permettre un mouvement qui à son tour aura pour résultat de

${ }^{33}$ Idem.

${ }^{34}$ Ibid., p. 65. 
nouvelles possibilités. Il nomme une telle avancée approfondissement ${ }^{35}$, qu'il définit comme un dépassement qui se maintient au sein de ce qu'il dépasse, qui donc creuse plus profond en lui (comme la plante qui, sans se déplacer, sans aller ailleurs, se meut pourtant vers toujours plus de profondeur et s'enracine), exactement comme l'homme qui pratique avance dans ses possibilités sans en choisir d'autres.

\section{Phénoménologie de la possibilité}

Cependant, cette naissance même du possible au cœur du mouvement transforme la nature même de ce que l'on entend par possibilité. Patočka distingue en effet « intentions motrices », " quasi-intentions de mouvements » et «intentions de quasi-mouvements ${ }^{36}$. Il définit ensuite les expressions qu'il emploie : « La quasi-intention est l'intention en tant que réflexion sur le mouvement, imagination du mouvement propre ou de celui d'un autre ; l'intention d'un quasi-mouvement est l'intention d'un mouvement représenté, du mouvement en tant qu'objet mental », alors que l'intention motrice est « [...] d'ores et déjà, le mouvement lui-même ». Il faut comprendre la quasi-intention de mouvement comme pensée d'un mouvement sans plus, du dehors, comme lorsque l'on réfléchit à un mouvement qui a été effectué par quelqu'un d'autre ou par nous-mêmes, mais sans que cette pensée du mouvement ne contienne la moindre intention de le reproduire : le « quasi- » irréalise l'intention elle-même, montre qu'il ne s'agit pas d'une possibilité dont on se charge vraiment, on joue avec cette possibilité comme possibilité. Avec l'intention de quasi-mouvement, au contraire, on passe de l'intention de représentation d'un mouvement à l'intention d'un mouvement représenté. L'intention de quasi-mouvement est une véritable intention, au sens où celui qui a une intention de quasi-mouvement prétend activement à la réalisation du mouvement dont elle est l'anticipation. Il subsiste cependant un écart entre l'intention et le mouvement : l'intention est encore abstraite, elle ignore ce que cela fait réellement et en première personne que de faire ce mouvement. On pourrait parler ici, pour reprendre une distinction sur laquelle Claudia Serban $^{37}$ attire notre attention à partir des Concepts fondamentaux de la

\footnotetext{
35 «L'élan qui nous porte vers le monde, notre ouverture au monde, conduit ainsi à de nouvelles formations matérielles, à de nouvelles objectités qui à leur tour rendent possible une guise nouvelle de notre propre vie, un nouvel approfondissement de nous mêmes », J. Patočka, « Leçons sur la corporéité », Papiers phénoménologiques, op. cit., p. 71.

${ }^{36} \mathrm{Ibid} .$, p. 73.

${ }^{37}$ C. Serban, "Capacités de l'animal, potentialités de l'ustensile et possibilités du Dasein », in Philosophie n 116, 2013/1, p. 32-47. (Voir aussi son ouvrage : Phénoménologie de la possibilité. Husserl et Heidegger, Paris, PUF, 2016).
} 
métaphysique de Heidegger, d'une possibilité sans capacité, c'est-à-dire, selon Patočka, d'une possibilité déconnectée d'un « je peux », d'un " je sais faire » et qui oublie donc le corps comme sa condition de possibilité (et combien de fois avons nous, en observant un mouvement réalisé de l'extérieur, pensé qu'il nous suffirait de le reproduire, qu'au fond, cela ne doit pas être si compliqué de faire ce que font ceux qui savent y faire, avant d'être démentis lamentablement par la maladresse de notre corps et notre manque d'habitude?).

L'intention motrice, en revanche, est une "possibilité qui n'est pas simplement possible ${ }^{38}$, c'est-à-dire qu'elle n'est pas un irréel irréel, mais un irréel réel, elle est chargée et prête à faire feu, une anticipation qui a un lien fort avec sa réalisation, parce qu'elle a elle-même été conquise depuis le mouvement : elle mord toujours déjà par là, malgré sa potentialité, sur le monde. Dire par conséquent que l'intention motrice réelle est le mouvement lui-même ne signifie pas qu'elle ne peut exister sans être actualisée dans un mouvement, mais qu'elle s'origine dans ce cercle du mouvement que nous avons mis en évidence plus haut, qu'elle vient du mouvement. Approfondir notre pénétration du monde revient très exactement à convertir sans cesse des intentions de quasi-mouvement en intentions motrices depuis les intentions motrices qui sont déjà nôtres. En d'autres termes, les possibilités concrètes ou intentions motrices, que l'on peut nommer à présent capacités, ne sont pas projetées volontairement (et donc abstraitement) par le Dasein, elles lui adviennent au cœur du mouvement et par le mouvement. Et c'est parce que nos capacités sont gorgées des mouvements qui les ont vu naître et les enveloppent encore comme des sédiments déposés constituant leur profondeur et leur densité, qu'elles sont engagées dans le monde et se donnent comme concrètes. C'est ainsi qu'il faut comprendre que les intentions motrices, bien qu'elles ne soient pas encore réalisées (elles ne sont pas remplies par le mouvement réel) sont d'ores et déjà le mouvement: par exemple, le soldat qui sait ce que cela fait que de faire la guerre peut tout à fait envisager la possibilité d'une guerre avec toute la concrétude et la richesse nécessaire, sans pour autant devoir à nouveau effectivement être placé dans la situation réelle d'un conflit armé.

On mesure donc à quel point Patočka bouleverse l'analytique existentiale heideggérienne. Pour Heidegger, le Dasein est l'instance où s'origine le possible (Entwerfendes von Möglichkeiten), qui réalise le jet en avant du pro-jet, envoi qui est l'événement fondamental du monde. C'est pourquoi le projet lui-même est « la structure primordiale

38 J. Patočka, « Leçons sur la corporéité », Papiers phénoménologiques, op. cit., p. 65. 
La pratique chez Patočka : une description phénoménologique...

de l'événement fondamental [...] qu'est la configuration du monde (Weltbildung) ${ }^{39}$. Or, pour Patočka, les intentions seulement pro-jetées sont des intentions de quasi-mouvement, des possibilités simplement possibles et abstraites parce qu'elles ne viennent pas de notre commerce avec le monde; en un mot, elles sont des velléités. Une vraie possibilité, pour Patočka, s'impose à nous, nous ne pouvons demeurer libres à son égard. Il écrit : «[...] elles [les possibilités] ne deviennent miennes qu'en se m'appropriant au même titre que je me les approprie - dans la réalisation $»^{40}$. Le renversement de la pronominalité indique ici que nous sommes l'objet des possibilités qui sont pourtant nos objets d'appropriation. Conquérir une possibilité c'est indissociablement agir et pâtir, c'est devenir l'objet de notre objet : une ekstase certes, mais aussi un recueillement. On se fait aux possibilités, on ne les fait pas ; mieux encore, il faut dire qu'une possibilité n'est mienne qu'à la condition de n'être pas de mon fait. C'est pourquoi le lexique du « savoir-faire », de l'aptitude, de la « capacité » qu'emploie Patočka, et qui désigne les possibilités advenues depuis notre séjour auprès des choses, est directement dirigé contre Heidegger qui oppose précisément la capacité vitale et la possibilité existentiale : «Le $\lambda$ ó $\gamma \mathrm{os}$ est un pouvoir (Vermögen), c'est-à-dire, en soi-même, le fait de disposer d'un rapport de soi à l'étant en tant que tel. À la différence de ceci, nous avons appelé aptitude (Fähigkeit) la possibilité de se comporter, d'être rapporté en étant pris et emporté $»^{41}$.

C'est donc le Verstand lui-même qui est remis en question par Patočka : il n'est plus envoi du possible et dessin du monde, mais saisie de ce que je n'ai pas créé moi-même ${ }^{42}$. La révélation du possible est un événement, comme si quelque chose se débloquait, tombait d'un ciel trop vaste pour notre propre vision, comme si «des écailles nous tombaient des yeux »: la résistance, le surcroît seulement encore présenti, tombe. D'un seul coup, le sens descend, nous échoit, et l'on comprend. Si bien qu'il faudrait ici reprendre la catégorie d'événemential, que l'on doit à Claude Romano, pour thématiser la compréhension ${ }^{43}$. En effet, Romano distingue l'existential comme mode d'être du Dasein, dont il est responsable et qu'il ouvre de l'intérieur (ici la

\footnotetext{
${ }^{39}$ M. Heidegger, Les concepts fondamentaux de la métaphysique. Monde, finitude, solitude, Paris, Gallimard, 1992, § 76, p. 517.

${ }^{40}$ J. Patočka, "[Corps, possibilités, monde, champ d'apparition]», Papiers phénoménologiques, op. cit., p. 118.

${ }^{41}$ Fähigkeit (aptitude ou capacité) s'oppose ici au Vermögen (le pouvoir). M. Heidegger, Les concepts fondamentaux de la métaphysique, op. cit., § 73, p. 485.

${ }^{42}$ J. Patočka, Papiers phénoménologiques, "[Corps, possibilités, monde, champ d'apparition]", op. cit., p. 118.

${ }^{43} \mathrm{Cl}$. Romano, L'événement et le monde, Paris, PUF, 1988.
} 
compréhension comme existential renvoie à l'ouverture par le Dasein de ses propres possibilités, de la possibilisation dont il est l'origine et le principe) de l'événemential comme ce qui s'ouvre dans le Dasein sans venir du Dasein lui-même. L'événemential advient de l'extérieur au Dasein sans toutefois lui être extérieur (ce qui arrive le modifie, ainsi se fond dans la texture même de son être). Or il semble bien que la compréhension chez Patočka soit en un sens éminent ce que Romano appelle un « événemential »: «L'événement au sens événemential ne s'inscrit pas dans le monde, mais ouvre un monde pour l'advenant ${ }^{44}$, l'événement événemential n'est donc pas une chose du monde, un mouvement dans le monde (un tremblement de terre, une déclaration de guerre), mais une transmutation du sujet qu'elle ouvre sur une autre dimension du monde. Nous arrivons donc aux termes de la phénoménologie patočkienne de la pratique : elle nous permet de penser une possibilisation événementiale depuis un mouvement subjectif dont les conditions de possibilité sont aussi les résultats, et qui aboutit au décèlement d'intentions motrices authentiques depuis la conversion de ces intentions vides que sont les intentions de quasimouvement.

\section{Conclusion : corporéité existentiale et appartenance au monde}

Il est donc clair que Patočka refuse l'intellectualisme qui grève l'analyse phénoménologique, tout en refusant la réification de la conscience : il s'agit donc de penser le sujet depuis le monde sans le faire tomber dans le monde (il est même probable que le seul moyen de déréifier la conscience, soit précisément de la réinscrire dans le monde, de la réaliser, pour qu'elle puisse devenir). Nous pouvons tirer de cette tentative plusieurs conclusions.

Tout d'abord, Patočka parvient bien à penser l'existence du Dasein depuis cette dépendance ontologique réciproque que nous avons nommée appartenance. En effet, puisque la possibilisation du Dasein est événementiale, qu'elle lui advient en se fondant pourtant en lui dans le mouvement, ce sont donc précisément les possibilités constitutives de la profondeur du monde qui passent en lui, auxquelles il doit se faire (les possibilités «se m'approprient»). Mais ce passage lui-même ne serait pas possible sans l'effort du Dasein qui se meut pour réaliser ce «je peux " déjà acquis. Patočka nomme "ajointement», "correspondance » ou « adhésion » (Sich-Fügen) ce rapport au monde :

${ }^{4}$ Ibid., p. 56. 
La pratique chez Patočka : une description phénoménologique...

Le mouvement subjectif semble ainsi présupposer deux éléments : un déterminant, un sujet qui vit dans des possibilités, en fait le projet, y effectue un choix afin de se déterminer soi-même, c'est-à-dire de modifier son état; et une correspondance dans le monde, au sein de l'étant, quelque chose qui s'accorde avec et obéit à la libre détermination du sujet ${ }^{45}$.

On voit ici la réciprocité : l'effort du sujet allant vers lui-même réalise et fait apparaître le monde, mais les possibilités mêmes qu'il réalise viennent du monde et sont conquises en lui. Le mouvement du sujet est ainsi le relais du mouvement du monde, exactement comme dans le maniement la main ne dépasse l'objet que vers lui-même et le fait éclore à soi : «[...] la main ne modifie en rien ce qu'elle manie ; la chose reste ce qu'elle était, ou plutôt devient, grâce au maniement, ce qu'elle est, ce dont elle recelait la disposition, la possibilité ${ }^{46}$.

Une telle conception de l'existence signifie que le corps propre n'est plus seulement le réquisit négatif de la subjectivité, une simple ressource, mais la source même de l'existence. L'existence jaillit ici depuis le corps pris en tant que témoin ontologique de notre identité d'être avec le monde. Néanmoins, notre phénoménologie de la pratique nous permet de donner une certaine transcendance au corps, c'est-à-dire de faire du corps la matière même de notre subjectivité sans toutefois la réifier. Patočka écrit incidemment que le corps rend possible le mouvement alors que le mouvement constitue en retour le $\operatorname{corps}^{47}$, ce qui signifie précisément que le corps est pris dans ce cercle du mouvement, dont les résultats sont aussi les conditions de possibilités. En d'autres termes, il est déposé par le mouvement qu'il engendre, c'est-à-dire qu'il se donne comme un être constituant constitué par cela même qu'il constitue. Cela signifie que d'un côté je suis mon corps, c'est-à-dire cet horizon de possibilités présentes à même les choses et grâce auxquelles elles apparaissent comme dotées d'un sens, mais qu'en même temps ce corps qui est mon corps est plus que mon corps, qu'il est aussi la possibilité originaire d'aller au-delà des possibilités $q u$ 'il est, il est auto-dépassement de soi, et donc transcendance (ainsi, en gymnastique, de la souplesse : tout individu a un niveau de souplesse donné, c'est-à-dire la capacité d'accomplir certains mouvements, mais nous savons tous qu'il est possible de devenir plus souple, seulement nous ne savons pas ce que cela signifie en propre que d'être capable de ce surcroît d'élasticité corporelle). Or, cet auto-dépassement qui confère

\footnotetext{
45 J. Patočka, Papiers phénoménologiques, op. cit., p. 26.

${ }^{46} \mathrm{~J}$. Patočka, Qu'est-ce que la phénoménologie ?, op. cit., p. 194.

${ }^{47}$ J. Patočka, Papiers phénoménologiques, "Phénoménologie et métaphysique du mouvement", op. cit., p. 22.
} 
au corps une subjectivité et rend possible la pratique, est la manifestation phénoménologique de cette appartenance au monde dont mon corps est le sceau, corps qui est toujours plus que mon corps, car il est $d u$ monde. 